Pacific

Journal of

Mathematics

\title{
A NEW GENERAL CONJUGATE BAILEY PAIR
}

Michael J. RowelL 


\title{
A NEW GENERAL CONJUGATE BAILEY PAIR
}

\author{
MiCHAEL J. ROWELL
}

\begin{abstract}
We introduce a new general conjugate Bailey pair which bridges the gap between Bailey and Slater's work and the work done recently by Andrews and Warnaar. With this new general pair we are able to find many useful conjugate Bailey pairs similar to those of Andrews and Warnaar. Using our new pairs we show results related to the sums of triangular numbers, indefinite quadratic forms and partition identities. We close with a brief discussion of the many other paths that can and will be taken in the future.
\end{abstract}

\section{Introduction}

The Bailey transform. In [1948], W. N. Bailey introduced a new proof of the Rogers-Ramanujan identities

$$
\sum_{n=0}^{\infty} \frac{q^{n^{2}}}{(q)_{n}}=\frac{1}{\left(q, q^{4} ; q^{5}\right)_{\infty}} \quad \text { and } \quad \sum_{n=0}^{\infty} \frac{q^{n(n+1)}}{(q)_{n}}=\frac{1}{\left(q^{2}, q^{3} ; q^{5}\right)_{\infty}}
$$

where we use the standard hypergeometric $q$-series notation [Gasper and Rahman 2004, page xvi]: For $|q|<1$,

$$
\begin{aligned}
& (a)_{k}=(a ; q)_{k}=(1-a)(1-a q) \cdots\left(1-a q^{k-1}\right)=\prod_{i=0}^{k-1}\left(1-a q^{i}\right) \\
& (a)_{\infty}=(a ; q)_{\infty}=\lim _{k \rightarrow \infty}(a ; q)_{k}=\prod_{i=0}^{\infty}\left(1-a q^{i}\right) \\
& \left(a_{1} ; q\right)_{k}\left(a_{2} ; q\right)_{k} \cdots\left(a_{n} ; q\right)_{k}=\left(a_{1}, a_{2}, \ldots, a_{n} ; q\right)_{k} .
\end{aligned}
$$

Bailey also included more Rogers-Ramanujan-type identities, which he had found using a similar method of proof. Two years later Bailey formulated this method into what is now known as the Bailey transform:

MSC2000: 05A19, 11P82, 05A18, 33D15.

Keywords: basic hypergeometric series, conjugate Bailey pairs. 
Theorem 1.1 (The Bailey transform). If

$\beta_{n}=\sum_{r=0}^{n} \alpha_{r} u_{n-r} v_{n+r} \quad$ and $\quad \gamma_{n}=\sum_{r=n}^{\infty} \delta_{r} u_{r-n} v_{r+n}, \quad$ then $\sum_{n=0}^{\infty} \alpha_{n} \gamma_{n}=\sum_{n=0}^{\infty} \beta_{n} \delta_{n}$, subject to conditions on the four sequences $\alpha_{n}, \beta_{n}, \gamma_{n}$ and $\delta_{n}$ which make all the infinite series absolutely convergent.

The main result of the Bailey transform is dependent on two relations. The first relation defines a Bailey pair, $\left(\alpha_{n}, \beta_{n}\right)$, and the second defines a conjugate Bailey pair, $\left(\delta_{n}, \gamma_{n}\right)$. With the introduction of the Bailey transform, Bailey included many general pairs of both types. We recall the following conjugate Bailey pair.

Corollary 1.2 [Bailey 1948]. If we let $u_{n}=1 /(q)_{n}$ and $v_{n}=1 /(a q)_{n}$ in the Bailey transform, then we have the conjugate Bailey pair

$$
\delta_{n}=\left(\rho_{1}\right)_{n}\left(\rho_{2}\right)_{n}\left(\frac{a q}{\rho_{1} \rho_{2}}\right)^{n}
$$

and

$$
\gamma_{n}=\frac{\left(a q / \rho_{1}\right)_{\infty}\left(a q / \rho_{2}\right)_{\infty}}{(a q)_{\infty}\left(a q / \rho_{1} \rho_{2}\right)_{\infty}} \cdot \frac{\left(\rho_{1}\right)_{n}\left(\rho_{2}\right)_{n}}{\left(a q / \rho_{1}\right)_{n}\left(a q / \rho_{2}\right)_{n}}\left(\frac{a q}{\rho_{1} \rho_{2}}\right)^{n} .
$$

The above conjugate Bailey pair was then used with the Bailey transform to show multiple Rogers-Ramanujan-type identities. The same conjugate Bailey pair was then used three years later by Slater [1952] to prove her list of around 130 new and known Rogers-Ramanujan-type identities.

1.1. The bilateral Bailey transforms. Andrews and Warnaar [2007] recently introduced a handful of new conjugate Bailey pairs. We state the pairs they found in the following variations of the Bailey transform (see their paper for proofs):

Theorem 1.3 (Symmetric bilateral Bailey transform). If

$\beta_{n}=\sum_{r=-n}^{n} \alpha_{r} u_{n-r} v_{n+r}$ and $\gamma_{n}=\sum_{r \geq|n|} \delta_{r} u_{r-n} v_{r+n}$, then $\sum_{n=-\infty}^{\infty} \alpha_{n} \gamma_{n}=\sum_{n=0}^{\infty} \beta_{n} \delta_{n}$, subject to conditions on the four sequences which make all of the relevant infinite series absolutely convergent.

Theorem 1.4 (Asymmetric bilateral Bailey transform). Let $m=\max \{n,-n-1\}$. If

$\beta_{n}=\sum_{r=-n-1}^{n} \alpha_{r} u_{n-r} v_{n+r+1}$ and $\gamma_{n}=\sum_{r=m}^{\infty} \delta_{r} u_{r-n} v_{r+n+1}$, then $\sum_{n=-\infty}^{\infty} \alpha_{n} \gamma_{n}=\sum_{n=0}^{\infty} \beta_{n} \delta_{n}$, subject to conditions on the four sequences which make all of the relevant infinite series absolutely convergent. 
We can now introduce the conjugate Bailey pairs of Andrews and Warnaar.

Theorem 1.5 [Andrews and Warnaar 2007]. Let $u_{n}=v_{n}=1 /\left(q^{2} ; q^{2}\right)_{n}$ in the symmetric bilateral Bailey transform. Then we have the conjugate Bailey pairs

$$
\delta_{n}=\frac{\left(q^{2} ; q^{2}\right)_{2 n}}{(-q ; q)_{2 n+1}} q^{n}, \quad \gamma_{n}=q^{-n^{2}} \sum_{j \geq|n|} q^{j^{2}+j}
$$

and

$$
\delta_{n}=(q)_{2 n} q^{n}, \quad \gamma_{n}=q^{-2 n^{2}} \sum_{j \geq 2|n|} q^{j(j+1) / 2} .
$$

Theorem 1.6 [Andrews and Warnaar 2007]. Let $u_{n}=v_{n}=1 /\left(q^{2} ; q^{2}\right)_{n}$ in the asymmetric bilateral Bailey transform. Then we have the conjugate Bailey pairs

$$
\delta_{n}=\frac{\left(q^{2} ; q^{2}\right)_{2 n+1}}{(-q ; q)_{2 n+2}} q^{n}, \quad \gamma_{n}=q^{-n(n+1)} \sum_{j \geq m} q^{j(j+2)}
$$

and

$$
\delta_{n}=(q)_{2 n+1} q^{n}, \quad \gamma_{n}=q^{-2 n(n+1)} \sum_{j \geq 2 m} q^{j(j+3) / 2},
$$

where $m=\max \{n,-n-1\}$.

One of the more striking observations of these new pairs is the existence of a restricted sum in $\gamma_{n}$, a characteristic not commonly seen in previous conjugate Bailey pairs. Andrews and Warnaar were then able to apply these pairs to show many results both new and known relating to false and partial theta series.

1.2. Bridging gaps and contributions. This work below will bridge the previously unknown gap between the work done by Bailey and Slater and the work done by Andrews and Warnaar. Section 2 introduces a new conjugate Bailey pair that encompasses the pairs used by Bailey and Slater and those used by Andrews and Warnaar.

In Section 3 we use our general theorem to define specific new conjugate Bailey pairs. In Sections 4-6 we touch on some of the many applications that are obtainable with these new conjugate Bailey pairs.

\section{A general conjugate Bailey pair}

In this section we introduce a new general conjugate Bailey pair. As we show in Section 3, its special cases tie together the conjugate Bailey pairs of Andrews and Warnaar as well as the conjugate Bailey pair used by Bailey and Slater. In finding our generalization, we are able to find many other new conjugate Bailey 
pairs similar to those of Andrews and Warnaar. Their applications are seen in later sections.

The following theorem is our main result regarding conjugate Bailey pairs. We present a very general conjugate Bailey pair and its proof. For the purpose of the proof, we define an ${ }_{n+1} \phi_{n}$ basic hypergeometric series as (see [Gasper and Rahman 2004, page 4])

$$
{ }_{n+1} \phi_{n}\left[\begin{array}{l}
a_{1}, \ldots, a_{n+1} \\
b_{1}, \ldots, b_{n}
\end{array} ; q, z\right]=\sum_{k=0}^{\infty} \frac{\left(a_{1}, \ldots, a_{n+1} ; q\right)_{k}}{\left(q, b_{1}, \ldots, b_{n} ; q\right)_{k}} z^{k} .
$$

Theorem 2.1. Let $u_{n}=1 /(q)_{n}$ and $v_{n}=1 /(f q)_{n}$. Then

$$
\delta_{n}=\frac{\left(e f q^{2} / a b c, e f q / a ; q\right)_{\infty}}{\left(e f q^{2} / a b, e f q^{2} / a c ; q\right)_{\infty}} \cdot \frac{(a, b, c ; q)_{n}}{(e q ; q)_{n}}\left(\frac{e f q^{2}}{a b c}\right)^{n}
$$

is a conjugate Bailey pair, and

$$
\begin{aligned}
\gamma_{n} & =\frac{(e f q / a, a ; q)_{n}}{(f q, f q / a ; q)_{n}}\left(-\frac{1}{a}\right)^{n} q^{-n(n-1) / 2} \\
& \times \sum_{j \geq n} \frac{\left(e f q^{n+1} / a, f q / a, b, c ; q\right)_{j}(e q / a ; q)_{j-n}}{\left(e f q^{2} / a b, e f q^{2} / a c, f q^{n+1}, e q ; q\right)_{j}(q ; q)_{j-n}}\left(1-e f q^{2 j+1} / a\right)\left(-\frac{e f}{b c}\right)^{j} q^{j(j+3) / 2} .
\end{aligned}
$$

Proof. Our proof is an application of Watson's ${ }_{8} \phi_{7}$ transformation:

$$
\begin{aligned}
& \gamma_{n}= \sum_{j \geq n} \frac{\delta_{j}}{(q ; q)_{j-n}(f q ; q)_{j+n}} \\
&= \frac{\left(e f q^{2} / a b c, e f q / a ; q\right)_{\infty}}{\left(e f q^{2} / a b, e f q^{2} / a c ; q\right)_{\infty}} \sum_{j \geq n} \frac{(a, b, c ; q)_{j}}{(e q ; q)_{j}(q ; q)_{j-n}(f q ; q)_{j+n}}\left(\frac{e f q^{2}}{a b c}\right)^{j} \\
&= \frac{\left(e f q^{2} / a b c, e f q / a ; q\right)_{\infty}}{\left(e f q^{2} / a b, e f q^{2} / a c ; q\right)_{\infty}} \frac{(a, b, c ; q)_{n}}{(e q ; q)_{n}(f q ; q)_{2 n}}\left(\frac{e f q^{2}}{a b c}\right)^{n}{ }_{3} \phi_{2}\left[\begin{array}{c}
a q^{n}, b q^{n}, c q^{n} \\
e q^{n+1}, f q^{2 n+1}
\end{array} ; q, \frac{e f q^{2}}{a b c}\right] \\
&= \frac{\left(e f q^{2} / a b c, e f q / a ; q\right)_{\infty}}{\left(e f q^{2} / a b, e f q^{2} / a c ; q\right)_{\infty}} \frac{(a, b, c ; q)_{n}}{(e q ; q)_{n}(f q ; q)_{2 n}}\left(\frac{e f q^{2}}{a b c}\right)^{n} \frac{\left(e f q^{n+2} / a b, e f q^{n+2} / a c ; q\right)_{\infty}}{\left(e f q^{2 n+2} / a, e f q^{2} / a b c ; q\right)_{\infty}} \\
& \times \lim _{d \mapsto \infty} \phi_{7} X,
\end{aligned}
$$

where

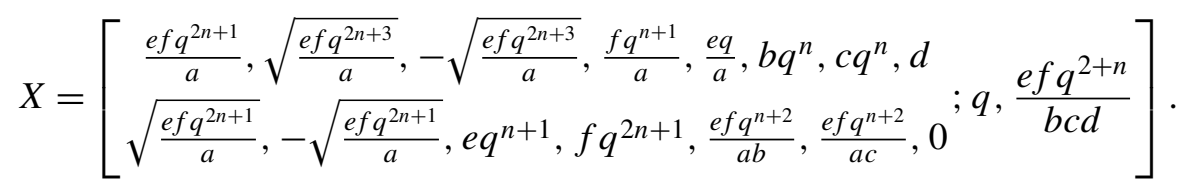

In the above, the last equality follows from [Gasper and Rahman 2004, Equation (III.17)] with $a=e f q^{2 n+1} / a, \quad b=f q^{n+1} / a, \quad c=e q / a, \quad d=q^{-k}, e=b q^{n}$ and $f=c q^{n}$, followed by $k \rightarrow \infty$. We note that allowing $d=q^{-k}$ ensures the 
termination of our series. After some simplification we see that this is

$$
\begin{aligned}
\gamma_{n}= & \frac{(e f q / a ; q)_{2 n+1}}{\left(e f q^{2} / a b, e f q^{2} / a c ; q\right)_{n}} \cdot \frac{(a, b, c ; q)_{n}}{(e q ; q)_{n}(f q ; q)_{2 n}}\left(\frac{e f q^{2}}{a b c}\right)^{n} \\
& \left.\times \lim _{d \mapsto \infty} \sum_{j=0}^{\infty} \frac{\left(1-\frac{e f q^{2 n+2 j+1}}{a}\right)\left(\frac{e f q^{2 n+1}}{a}, \frac{f q^{n+1}}{a}, \frac{e q}{a}, b q^{n}, c q^{n}, d ; q\right)_{j}}{\left(1-\frac{e f q^{2 n+1}}{a}\right)\left(e q^{n+1}, f q^{2 n+1}, \frac{e f q^{n+2}}{a b}, \frac{e f q^{n+2}}{a c}, q ; q\right)_{j}}\right)^{j} \\
= & \frac{(e f q / a, a ; q)_{n}}{(f q, f q / a ; q)_{n}}\left(-\frac{1}{a}\right)^{n} q^{-n(n-1) / 2} \\
& \times \sum_{j \geq n} \frac{\left(e f q^{n+1} / a, f q / a, b, c ; q\right)_{j}(e q / a ; q)_{j-n}}{\left(e f q^{2} / a b, e f q^{2} / a c, f q^{n+1}, e q ; q\right)_{j}(q ; q)_{j-n}}\left(1-e f q^{2 j+1} / a\right)\left(-\frac{e f}{b c}\right)^{j} q^{j(j+3) / 2},
\end{aligned}
$$

the desired expression.

We note that our conjugate Bailey pair presented above has the form

$$
\gamma_{n}=C_{n} \sum_{j=n}^{\infty} D_{j} \frac{(e q / a)_{j-n}(e f q / a)_{j+n}}{(q)_{j-n}(f q)_{j+n}} .
$$

Definition 2.2 [Andrews 2001]. Two sequences $\alpha_{n}(A, K)$ and $\beta_{n}(A, K)$ form a WP-Bailey pair if

$$
\beta_{n}(A, K)=\sum_{j=0}^{n} \frac{(K / A)_{n-j}(K)_{n+j}}{(q)_{n-j}(A q)_{n+j}} \alpha_{j}(A, K) .
$$

Andrews uses this definition to define the WP-Bailey chain. In the same spirit, we can define our own WP-conjugate Bailey pair:

Definition 2.3. We say that two sequences $\delta_{n}(A, K)$ and $\gamma_{n}(A, K)$ form a $W P$ conjugate Bailey pair if

$$
\gamma_{n}(A, K)=\sum_{j=n}^{\infty} \frac{(K / A)_{n-j}(K)_{n+j}}{(q)_{n-j}(A q)_{n+j}} \delta_{j}(A, K) .
$$

We can then see that Theorem 2.1 satisfies such a definition if we choose the two sequences $\left(\gamma_{n} / C_{n}, D_{n}\right)$ with $A=f$ and $K=e f q / a$. We will not explore the realm of WP-conjugate Bailey chains here, but we certainly foresee its appearance in subsequent work.

\section{Specific conjugate Bailey pairs}

The conjugate Bailey pair of Corollary 1.2, used by Bailey [1948] and Slater [1952] in their work, is a special case of our theorem. We can see this by allowing $a=e q$, followed by some simple change of variables. We also note that the special case 
$a=e q$ in Theorem 2.1 not only simplifies our pair but also completely eliminates the restricted sum from $\gamma_{n}$. To recover the pairs found by Andrews and Warnaar, we explore another option that will simplify the term $(e q / a ; q)_{j-n} /(q ; q)_{j-n}$ in our $\gamma_{n}$. We can accomplish this by allowing $e \rightarrow a$. We will consider two corollaries of our main result:

Corollary 3.1. Let $u_{n}=v_{n}=1 /\left(q^{2} ; q^{2}\right)_{n}$ in the Bailey transform. Then we have the conjugate Bailey pair

$$
\begin{aligned}
\gamma_{n}=\frac{\left(a ; q^{2}\right)_{n}}{\left(q^{2} / a ; q^{2}\right)_{n}}\left(-\frac{1}{a}\right)^{n} q^{-n(n-1)} & \\
& \times \sum_{j \geq n} \frac{\left(q^{2} / a, b, c ; q^{2}\right)_{j}}{\left(q^{4} / b, q^{4} / c, a q^{2} ; q^{2}\right)_{j}}\left(1-q^{4 j+2}\right)\left(-\frac{a}{b c}\right)^{j} q^{j(j+3)}
\end{aligned}
$$

and

$$
\delta_{n}=\frac{\left(q^{4} / b c, q^{2} ; q^{2}\right)_{\infty}}{\left(q^{4} / b, q^{4} / c ; q^{2}\right)_{\infty}} \cdot \frac{\left(a, b, c ; q^{2}\right)_{n}}{\left(a q^{2} ; q^{2}\right)_{n}}\left(\frac{q^{4}}{b c}\right)^{n} .
$$

Proof. Let $e \rightarrow a$ and $f=1$ and $q \rightarrow q^{2}$ in Theorem 2.1.

Corollary 3.2. Let $u_{n}=1 /\left(q^{2} ; q^{2}\right)_{n}$ and $v_{n}=1 /\left(q^{4} ; q^{2}\right)_{n}$ in the Bailey transform. Then we have the conjugate Bailey pair

$$
\begin{aligned}
\gamma_{n}=\frac{\left(a ; q^{2}\right)_{n}}{\left(q^{4} / a ; q^{2}\right)_{n}}\left(-\frac{1}{a}\right)^{n} q^{-n(n-1)} & \\
& \times \sum_{j \geq n} \frac{\left(q^{4} / a, b, c ; q^{2}\right)_{j}}{\left(q^{6} / b, q^{6} / c, a q^{2} ; q^{2}\right)_{j}}\left(1-q^{4 j+4}\right)\left(-\frac{a}{b c}\right)^{j} q^{j(j+5)}
\end{aligned}
$$

and

$$
\delta_{n}=\frac{\left(q^{6} / b c, q^{4} ; q^{2}\right)_{\infty}}{\left(q^{6} / b, q^{6} / c ; q^{2}\right)_{\infty}} \cdot \frac{\left(a, b, c ; q^{2}\right)_{n}}{\left(a q^{2} ; q^{2}\right)_{n}}\left(\frac{q^{6}}{b c}\right)^{n} .
$$

Proof. Let $e \rightarrow a$ and $f=q$ and $q \rightarrow q^{2}$ in Theorem 2.1.

We now have the proper tools to prove Andrews and Warnaar's results.

Proof of Theorem 1.5. When using the symmetric bilateral Bailey transform with $u_{n}=v_{n}=1 /\left(q^{2} ; q^{2}\right)_{n}$, we have $\gamma_{n}=\gamma_{-n}$, so that we can assume $n>0$. But then the relation between $\gamma$ and $\delta$ in Theorem 1.3 is the same as that of Theorem 1.1. It is left to show that the conjugate Bailey pairs due to Andrews and Warnaar are special cases of Corollary 3.1. We see this by considering $a=-q, b=q$ and $c=q^{2}$ in Corollary 3.1 and $b=q, c=q^{2}$ and $a \rightarrow 0$ in Corollary 3.1.

Proof of Theorem 1.6. When using the asymmetric bilateral Bailey transform with $u_{n}=v_{n}=1 /\left(q^{2} ; q^{2}\right)_{n}$, we see that

$$
\gamma_{n}=\sum_{j \geq m} \frac{\delta_{j}}{\left(q^{2} ; q^{2}\right)_{j-n}\left(q^{2} ; q^{2}\right)_{j+n+1}}=\sum_{j \geq m} \frac{\delta_{j}}{\left(q^{2} ; q^{2}\right)_{j-m}\left(q^{2} ; q^{2}\right)_{j+m+1}},
$$


where $m=\max \{n,-n-1\}$. If we consider $a=-q^{2}, b=q^{2}$ and $c=q^{3}$ in Corollary 3.2, we see that

$$
\delta_{n}=\frac{\left(q, q^{4} ; q^{2}\right)_{\infty}}{\left(q^{4}, q^{3} ; q^{2}\right)_{\infty}} \cdot \frac{\left(-q^{2}, q^{2}, q^{3} ; q^{2}\right)_{n}}{\left(-q^{4} ; q^{2}\right)_{n}}\left(\frac{q^{6}}{q^{5}}\right)^{n}=\frac{\left(1+q^{2}\right)}{\left(1+q^{2 n+2}\right)}(q)_{2 n+1} q^{n}
$$

and $\gamma_{n}$ is equal to

$$
\begin{aligned}
\frac{\left(-q^{2} ; q^{2}\right)_{n}}{\left(-q^{2} ; q^{2}\right)_{n}}\left(\frac{1}{q^{2}}\right)^{n} q^{-n(n-1)} \sum_{j \geq n} \frac{\left(-q^{2}, q^{2}, q^{3} ; q^{2}\right)_{j}}{\left(q^{4}, q^{3},-q^{4} ; q^{2}\right)_{j}} & \left(1-q^{4 j+4}\right)\left(\frac{q^{2}}{q^{5}}\right)^{j} q^{j(j+5)} \\
& =\left(1-q^{4}\right) q^{-n(n+1)} \sum_{j \geq n} q^{j(j+2)} .
\end{aligned}
$$

Thus,

$$
q^{-n(n+1)} \sum_{j \geq n} q^{j(j+2)}=\sum_{j \geq n} \frac{(q ; q)_{2 j+1} q^{j}}{\left(1+q^{2 j+2}\right)\left(q^{2} ; q^{2}\right)_{j-n}\left(q^{2} ; q^{2}\right)_{j+n+1}} .
$$

Since $n(n+1)=m(m+1)$ when $m=\max \{n,-n-1\}$, we are done.

We can prove the second pair in the same way using $b=q^{2}, c=q^{3}$ and $a \rightarrow 0$ in Corollary 3.2.

We now introduce some new special cases of our general conjugate Bailey pair; these will be used in later sections. All of the conjugate Bailey pairs we introduce below are with respect to the symmetric bilateral Bailey transform and $u_{n}=v_{n}$. This ensures that if $\gamma_{n}$ is a conjugate Bailey pair with respect to the Bailey transform, then $\gamma_{n}^{\prime}=\gamma_{|n|}$ is a conjugate Bailey pair with respect to the symmetric bilateral Bailey transform. Thus, each conjugate Bailey pair that satisfies Corollary 3.1 is a pair in the symmetric bilateral Bailey transform as well as the Bailey transform.

Corollary 3.3. Let $u_{n}=v_{n}=1 /\left(q^{2} ; q^{2}\right)_{n}$ in the symmetric bilateral Bailey transform. Then we have the conjugate Bailey pairs listed in Table 1.

Proof. Each pair follows from a choice of $a, b$ and $c$ in Corollary 3.1.

For (1), we take $a=b=c=q$, followed by $q \rightarrow-q$.

For (2), we take $a=-b=-c=q$, followed by $q \rightarrow-q$.

For (3), we take $a=b=q, c=-q^{2}$, followed by $q \rightarrow-q$.

For (4), we take $a=-q, b=q^{3}$ and $c \rightarrow \infty$.

For (5), we take $a=-b=q, c \rightarrow \infty$, followed by $q \rightarrow-q$.

For (6), we take $b=c=q, a \rightarrow 0$.

For (7), we take $b=q, a \rightarrow 0, c \rightarrow \infty$.

For (8), we take $b=q^{3}, a \rightarrow 0, c \rightarrow \infty$.

For (9), we take $b=q, c=-q^{2}, a \rightarrow 0$, followed by $q \rightarrow-q$.

For (10), we take $a=q, b=q^{2}$ and $c \rightarrow \infty$, followed by $q \rightarrow-q$. 


\begin{tabular}{|c|c|c|}
\hline & $\delta_{n}$ & $\gamma_{n}$ \\
\hline (1) & $\frac{\left(q^{2} ; q^{2}\right)_{\infty}^{2}}{\left(-q ; q^{2}\right)_{\infty}^{2}} \cdot \frac{\left(-q ; q^{2}\right)_{n}^{2}}{\left(1+q^{2 n+1}\right)} q^{2 n}$ & $q^{-n^{2}} \sum_{j>|n|} \frac{\left(1-q^{2 j+1}\right)}{\left(1+q^{2 j+1}\right)^{2}} q^{j(j+2)}$ \\
\hline (2) & $\frac{\left(q^{2} ; q^{2}\right)_{\infty}^{2}}{\left(q ; q^{2}\right)_{\infty}^{2}} \cdot \frac{\left(q ; q^{2}\right)_{n}^{2}}{\left(1+q^{2 n+1}\right)} q^{2 n}$ & $q^{-n^{2}} \sum_{j \geq|n|} \frac{q^{j(j+2)}}{\left(1-q^{2 j+1}\right)}$ \\
\hline (3) & $\frac{(q)_{\infty}}{(-q)_{\infty}} \cdot \frac{(-q)_{2 n}}{\left(1+q^{2 n+1}\right)} q^{n}$ & $q^{-n^{2}} \sum_{j>|n|} \frac{\left(1-q^{2 j+1}\right)}{\left(1+q^{2 j+1}\right)} q^{j(j+1)}$ \\
\hline (4) & $\frac{\left(q^{2} ; q^{2}\right)_{\infty}}{\left(q ; q^{2}\right)_{\infty}} \cdot \frac{\left(q ; q^{2}\right)_{n+1}}{\left(1+q^{2 n+1}\right)}(-1)^{n} q^{n^{2}}$ & $\left(1-q^{2 j+1}\right)^{2}(-1)^{j} q^{2 j^{2}}$ \\
\hline (5) & $\frac{\left(q^{2} ; q^{2}\right)_{\infty}}{\left(q ; q^{2}\right)_{\infty}} \cdot \frac{\left(q ; q^{2}\right)_{n}}{\left(1+q^{2 n+1}\right)}(-1)^{n} q^{n(n+2)}$ & $-n^{2} \sum(-1)^{j} q^{2 j(j+1)}$ \\
\hline (6) & $\frac{\left(q^{2} ; q^{2}\right)_{\infty}^{2}}{\left(q ; q^{2}\right)_{\infty}^{2}}\left(q ; q^{2}\right)_{n}^{2} q^{2 n}$ & $q^{-2 n^{2}} \sum_{j \geq|n|} \frac{\left(1+q^{2 j+1}\right)}{\left(1-q^{2 j+1}\right)} q^{2 j(j+1)}$ \\
\hline (7) & $\frac{\left(q^{2} ; q^{2}\right)_{\infty}}{\left(q ; q^{2}\right)_{\infty}}\left(q ; q^{2}\right)_{n}(-1)^{n} q^{n(n+2)}$ & $\sum\left(1+q^{2 j+1}\right)(-1)^{j} q^{j(3 j+2)}$ \\
\hline (8) & $\frac{\left(q^{2} ; q^{2}\right)_{\infty}}{\left(q ; q^{2}\right)_{\infty}}\left(q ; q^{2}\right)_{n+1}(-1)^{n} q^{n^{2}}$ & $q^{-2 n^{2}} \sum_{j \geq|n|}\left(1-q^{2 j+1}\right)\left(1-q^{4 j+2}\right)(-1)^{j} q^{3 j^{2}}$ \\
\hline (9) & $\frac{(q)_{\infty}}{(-q)_{\infty}}(-q)_{2 n} q^{n}$ & $q^{-2 n^{2}} \sum_{j>2 \mid n\rfloor}(-1)^{j} q^{j(j+1) / 2}$ \\
\hline (10) & $\frac{\left(q^{2} ; q^{2}\right)_{n}}{\left(1+q^{2 n+1}\right)}(-1)^{n} q^{n(n+1)}$ & $q^{-n^{2}} \sum_{j \geq 2|n|}(-q)^{j(j+1) / 2}$ \\
\hline
\end{tabular}

\section{Table 1}

Corollary 3.4. Let $u_{n}=v_{n}=1 /(q)_{n}$ in the symmetric bilateral Bailey transform. Then we have conjugate Bailey pairs listed in Table 2.

Proof. Again, each pair follows from a choice of $a, b$ and $c$ in Corollary 3.1.

For (11), we take $b=-c=q, a \rightarrow 0$, followed by $q^{2} \rightarrow q$.

For (12), we take $b=-q^{2}, a \rightarrow 0$ and $c \rightarrow \infty$, followed by $q^{2} \rightarrow q$.

For (13), we take $b=q^{2}, a \rightarrow 0$ and $c \rightarrow \infty$, followed by $q^{2} \rightarrow q$.

For (14), we take $a \rightarrow 0$ and $b, c \rightarrow \infty$, followed by $q^{2} \rightarrow q$.

\section{Sums of triangular numbers}

We turn our attention to Gauss's formula

$$
\sum_{n=0}^{\infty} q^{n(n+1) / 2}=\frac{\left(q^{2} ; q^{2}\right)_{\infty}}{\left(q ; q^{2}\right)_{\infty}}
$$




\begin{tabular}{|c|c|c|}
\hline & $\delta_{n}$ & $\gamma_{n}$ \\
\hline (11) & $\frac{\left(q^{2} ; q^{2}\right)_{\infty}}{\left(q ; q^{2}\right)_{\infty}}\left(q ; q^{2}\right)_{n}(-1)^{n} q^{n}$ & $q^{-n^{2}} \sum_{j \geq|n|}(-1)^{j} q^{j(j+1)}$ \\
\hline (12) & $\frac{(q)_{\infty}}{(-q)_{\infty}}(-q)_{n} q^{n(n+1) / 2}$ & $q^{-n^{2}} \sum_{j \geq|n|}\left(1-q^{2 j+1}\right) q^{j(3 j+1) / 2}$ \\
\hline (13) & $(q)_{n}(-1)^{n} q^{n(n+1) / 2}$ & $q^{-n^{2}} \sum\left(1-q^{2 j+1}\right)(-1)^{j} q^{j(3 j+1) / 2}$ \\
\hline (14) & $(q)_{\infty} q^{n(n+1)}$ & $q^{-n^{2}} \sum^{j-1}(-1)^{j} q^{j(j+1) / 2}$ \\
\hline
\end{tabular}

\section{Table 2}

and remark that $n(n+1) / 2$ is the $n$-th triangular number. With this identity, we are able to make some interesting remarks about generating functions related to the sums of the triangular numbers. We define the function $\psi(q)=\sum_{n=0}^{\infty} q^{n(n+1) / 2}$, a classical theta function studied by Ramanujan [Berndt 1991, page 100, (5.1)]. We can then see that $\psi^{k}(q)=\sum_{n=0}^{\infty} t_{k}(n) q^{n}$, where $t_{k}(n)$ counts the number of representations of $n$ as the sum of $k$ triangular numbers. We note that order is important, unlike with partitions: for example, $t_{3}(5)=3$ since $5=3+1+1=$ $1+3+1=1+1+3$. While (15)-(18) and (20) can be found in previous work, none have used the conjugate Bailey pair approach presented below, and no other method has been able to encompass so many results.

\section{Corollary 4.1.}

$$
\psi^{2}(q)=\sum_{j=-\infty}^{\infty} \frac{(-1)^{j} q^{j(j+1)}}{\left(1-q^{2 j+1}\right)},
$$

$$
\begin{aligned}
& \psi^{2}\left(q^{2}\right)=\sum_{j=0}^{\infty} \frac{q^{j}}{1+q^{2 j+1}}, \\
& \psi^{4}(q)=\sum_{j=-\infty}^{\infty} \frac{q^{j}}{\left(1-q^{2 j+1}\right)^{2}},
\end{aligned}
$$

$$
\psi^{3}(q)=\sum_{j \geq|n|} \frac{\left(1+q^{2 j+1}\right)}{\left(1-q^{2 j+1}\right)} q^{2 j(j+1)-n(2 n-1)},
$$$$
\psi^{2}(q)=\sum_{j \geq|n|}(-1)^{j+n} q^{j(3 j+2)-n(3 n+1)}\left(1+q^{2 j+1}\right),
$$

$$
\psi^{2}(q)=\sum_{j \geq|n|}(-1)^{j+n} q^{j(j+1)-n^{2}} .
$$


We note that (16) can be found in [Berndt 1991, page 139, Example (iv)], (17) can be found in [Dickson 1966, page 285], (18) can be found in [Andrews 1986a, page 114, (1.5)], and (20) can be found in [Andrews 1984, page 452, (1.4)] with $q \rightarrow q^{2}$ and $z=1 / q$.

Proof. For Equation (15), we consider the Bailey pair

$$
\alpha_{n}=q^{n^{2}}, \quad \beta_{n}=\frac{\left(-q ; q^{2}\right)_{n}^{2}}{\left(q^{2} ; q^{2}\right)_{2 n}}
$$

found in [Andrews 1998, page 49, Example 1]. Combining this Bailey pair with the conjugate Bailey pair (3) with $q \rightarrow-q$ and $u_{n}=v_{n}=1 /\left(q^{2} ; q^{2}\right)_{n}$, we get

$$
\begin{aligned}
& \sum_{n=-\infty}^{\infty} \frac{(-1)^{n} q^{n(n+1)}}{\left(1-q^{2 n+1}\right)}=\sum_{n=-\infty}^{\infty}(-1)^{n} \sum_{j \geq|n|} \frac{\left(1+q^{2 j+1}\right)}{\left(1-q^{2 j+1}\right)} q^{j(j+1)}
\end{aligned}
$$



where our last equality is due to [Gasper and Rahman 2004, III.2, page 359].

For Equation (16), we consider the Bailey pair

$$
\alpha_{n}=q^{n}, \quad \beta_{n}=\frac{q^{-n}}{(q)_{2 n}}
$$

found in [Slater 1951, F(3)] with the conjugate Bailey pair (2) with $q \rightarrow-q$ and $u_{n}=v_{n}=1 /\left(q^{2} ; q^{2}\right)_{n}$ to get

$$
\begin{aligned}
& \sum_{j=0}^{\infty} \frac{q^{j}}{1+q^{2 j+1}}=\sum_{n=-\infty}^{\infty}(-1)^{n} q^{-n(n-1)} \sum_{j \geq|n|} \frac{(-1)^{j} q^{j(j+1)}}{1+q^{2 j+1}} \\
& =\frac{\left(q^{2} ; q^{2}\right)_{\infty}^{2}}{(1-q)\left(-q ; q^{2}\right)_{\infty}^{2}}{ }_{2} \phi_{1}\left(\begin{array}{c}
-q,-q \\
\left.q^{3} ; q^{2}, q\right)
\end{array}\right. \\
& =\frac{\left(q^{2} ; q^{2}\right)_{\infty}^{2}}{\left(-q ; q^{2}\right)_{\infty}^{2}} \cdot \frac{\left(-q^{2} ; q^{2}\right)_{\infty}^{2}}{\left(q ; q^{2}\right)_{\infty}^{2}}
\end{aligned}
$$

where our first equality is due to the identity

$$
\sum_{n=-j}^{j}(-1)^{n} q^{-n(n-1)}=(-1)^{j} q^{-j(j+1)},
$$

which can be easily proved with induction; our last equality is due to [Gasper and Rahman 2004, III.2, page 359]. We then see that our infinite product is $\psi^{2}\left(q^{2}\right)$.

For Equation (17), we consider the Bailey pair (22) with the conjugate Bailey pair (1) with $u_{n}=v_{n}=1 /\left(q^{2} ; q^{2}\right)_{n}$. We then apply (23) and [Gasper and Rahman 2004, III.2, page 359] as with our previous result. 
For Equation (18), we consider the Bailey pair (22) with the conjugate Bailey pair (6) with $u_{n}=v_{n}=1 /\left(q^{2} ; q^{2}\right)_{n}$ to get

$$
\begin{aligned}
\sum_{j \geq|n|} \frac{\left(1+q^{2 j+1}\right)}{\left(1-q^{2 j+1}\right)} q^{2 j(j+1)-n(2 n-1)} & =\sum_{n=-\infty}^{\infty} q^{-n(2 n-1)} \sum_{j \geq|n|} \frac{\left(1+q^{2 j+1}\right)}{\left(1-q^{2 j+1}\right)} q^{2 j(j+1)} \\
& =\frac{\left(q^{2} ; q^{2}\right)_{\infty}^{2}}{\left(q ; q^{2}\right)_{\infty}^{2}} \sum_{n=0}^{\infty} \frac{\left(q ; q^{2}\right)_{n}^{2}}{(q)_{2 n}} q^{n} \\
& =\frac{\left(q^{2} ; q^{2}\right)_{\infty}^{2}}{\left(q ; q^{2}\right)_{\infty}^{2}} \cdot \frac{\left(q^{2} ; q^{2}\right)_{\infty}}{\left(q ; q^{2}\right)_{\infty}}
\end{aligned}
$$

where the last equality is due to $q$-binomial theorem [Andrews 1998, page 17, Theorem 2.1]

$$
\sum_{n=0}^{\infty} \frac{(a)_{n}}{(q)_{n}} t^{n}=\frac{(a t)_{\infty}}{(t)_{\infty}} .
$$

For Equation (19), we consider the Bailey pair

$$
\alpha_{n}=(-1)^{n} q^{-n(n+1)}, \quad \beta_{n}=\frac{(-1)^{n} q^{-n(n+1)}}{\left(q^{2} ; q^{2}\right)_{n}}
$$

found in the fourth row of the second table in [Slater 1951, page 468] with the conjugate Bailey pair (8) with $u_{n}=v_{n}=1 /\left(q^{2} ; q^{2}\right)_{n}$. We then apply the $q$-binomial theorem as with our previous result.

For Equation (20), we consider the Bailey pair

$$
\alpha_{n}=(-1)^{n}, \quad \beta_{n}=\frac{(-1)^{n}}{\left(q^{2} ; q^{2}\right)_{n}}
$$

found in the seventh row of the second table in [Slater 1951, page 468] with the conjugate Bailey pair (10) with $u_{n}=v_{n}=1 /(q)_{n}$. We then apply the $q$-binomial theorem as with our two previous results.

\section{Indefinite quadratic forms}

In the previous section we noticed that our new general conjugate Bailey pair is very capable of producing results of the form (18)-(20). In this section we take a deeper look into double series involving an indefinite quadratic form. By [1959], E. Hecke had studied many of these forms in detail, and among these was

$$
\sum_{j \geq 2|n|}(-1)^{j+n} q^{j(j+1) / 2-n(3 n-1) / 2}=(q)_{\infty}^{2},
$$

which was originally discovered by L. J. Rogers [1894]. 
It is with little difficulty that we can show the above identity and others with our new conjugate Bailey pairs. Noting that our $\gamma_{n}$ is already a restricted sum of the type we are looking for, all that is left is to find a suitable Bailey pair to match it with. The following section will discuss some new results as well as tying in some identities due to Andrews [1986a] and Andrews, Dyson, and Hickerson [1988].

Corollary 5.1. Identity (26) is true.

Proof. We consider the Bailey pair

$$
\alpha_{2 n}=(-1)^{n} q^{n(n+1)}, \alpha_{2 n+1}=0, \quad \beta_{n}=\frac{q^{n(n-1) / 2}}{(q)_{n}\left(q ; q^{2}\right)_{n}},
$$

which is found in [Slater 1951, C(5)], with the conjugate Bailey pair (10) in which $u_{n}=v_{n}=1 /(q)_{n}$. Applying (24) and allowing $q^{2} \rightarrow q$ yields our final result.

In [1986a], Andrews uses complicated Bailey pairs with the implementation of Bailey chains, as well as some clever algebra to prove identities such as the following.

\section{Corollary 5.2.}

$$
\sum_{j \geq|n|}(-1)^{n} q^{j(3 j+1) / 2-n^{2}}\left(1-q^{2 j+1}\right)=\frac{(q)_{\infty}^{2}}{(-q)_{\infty}} .
$$

Proof. We consider the Bailey pair (25) with the conjugate Bailey pair (11) with $u_{n}=v_{n}=1 /(q)_{n}$. Our result then follows with the application of (24).

In [1988], Andrews, Dyson and Hickerson then adapted the method used in [Andrews 1986a] to prove similar identities involving the rank of a partition. The rank of a partition is the excess of the largest part over the number of parts. The main motivation for their paper was the function

$$
\sigma(q)=\sum_{n=0}^{\infty} \frac{q^{n(n+1) / 2}}{(-q)_{n}},
$$

which can be found in [Andrews 1986b]. We note that $\sigma(q)$ is the generating function for strict partitions with odd rank subtracted from those with even rank. We find the following corollary in [Andrews et al. 1988, page 392, Equation (1.5)]. Corollary 5.3.

$$
\sigma(q)=\sum_{j \geq|n|}(-1)^{n+j} q^{j(3 j+1) / 2-n^{2}}\left(1-q^{2 j+1}\right) .
$$

Proof. We consider the Bailey pair (25) with the conjugate Bailey pair (12) with $u_{n}=v_{n}=1 /(q)_{n}$. 
Andrews, Dyson and Hickerson, in [1988, page 404], define generating a function similar to $\sigma(q)$, as follows. For $n \geq 1$, consider partitions of $n$ into odd parts, with the property that if $k$ occurs as a part, then all positive odd parts less than $k$ must also occur (without odd gaps). Let $S^{*}(n)$ be the excess of the number of such partitions with largest part congruent to 3 modulo 4 over the number with largest part congruent to 1 modulo 4. They then show [1988, page 404, Equation 5.2] that

$$
\sum_{n \geq 1} S^{*}(n) q^{n}=\sum_{n \geq 1} \frac{(-1)^{n} q^{n^{2}}}{\left(q ; q^{2}\right)_{n}}=\sum_{n \geq 1}(-1)^{n} q^{n(3 n+1)}\left(1+q^{2 n}\right) \sum_{j=0}^{2 n-1} q^{-j(j+1) / 2} .
$$

It is with minimal work that we can show an equivalent formula.

Corollary 5.4.

$$
\sum_{n \geq 0} \frac{(-1)^{n} q^{n^{2}}}{\left(q ; q^{2}\right)_{n}}=\sum_{j \geq|n|}(-1)^{j} q^{j(3 j+1)-n(2 n-1)}\left(1-q^{4 n+2}\right) .
$$

Proof. We consider the Bailey pair (22) with the conjugate Bailey pair (12) with $u_{n}=v_{n}=1 /\left(q^{2} ; q^{2}\right)_{n}$. Applying (24) yields our result.

The following identity can be found in [Andrews 1984, page 457, (3.16)],

\section{Corollary 5.5.}

$$
\sum_{j \geq 2|n|}(-1)^{j+n} q^{j(j+1) / 2-n^{2}}=(q)_{\infty}\left(q^{2} ; q^{2}\right)_{\infty}
$$

Proof. We consider the Bailey pair (25) with the conjugate Bailey pair (13) with $u_{n}=v_{n}=1 /(q)_{n}$.

The following corollaries are new indefinite quadratic forms.

\section{Corollary 5.6.}

$$
\begin{aligned}
& \sum_{j \geq|n|}(-1)^{j} q^{2 j(j+1)-n(2 n-1)}=\frac{\left(q^{4} ; q^{4}\right)_{\infty}^{2}}{\left(-q ; q^{2}\right)_{\infty}} \\
& \sum_{j \geq|n|}(-1)^{j} q^{j(3 j+2)-n(2 n-1)}\left(1+q^{2 j+1}\right)=\frac{\left(q^{2} ; q^{2}\right)_{\infty}^{2}}{\left(q ; q^{2}\right)_{\infty}},
\end{aligned}
$$

$$
\sum_{j \geq|n|}(-1)^{j} q^{3 j^{2}-n(2 n-1)}\left(1-q^{2 j+1}\right)^{2}\left(1+q^{2 j+1}\right)=-q \frac{\left(q^{2} ; q^{2}\right)_{\infty}^{2}}{\left(q ; q^{2}\right)_{\infty}},
$$

$$
\sum_{j \geq 2|n|}(-1)^{j+n} q^{j(j+1) / 2-2 n^{2}}=\frac{(q)_{\infty}^{2}}{\left(-q ; q^{2}\right)_{\infty}} .
$$

Proof. For (29) we consider the Bailey pair (22) with the conjugate Bailey pair (10) with $q \rightarrow q^{2}$ and $u_{n}=v_{n}=1 /\left(q^{2} ; q^{2}\right)_{n}$. The result then follows from (24). 
For (30) we consider the Bailey pair (22) with the conjugate Bailey pair (8) with $u_{n}=v_{n}=1 /\left(q^{2} ; q^{2}\right)_{n}$. Our result follows from applying (24).

For (31) we consider the Bailey pair (22) with the conjugate Bailey pair (9) with $u_{n}=v_{n}=1 /\left(q^{2} ; q^{2}\right)_{n}$. Our result follows from applying (24):

$$
\begin{aligned}
\sum_{j \geq|n|}( & -1)^{j} q^{3 j^{2}-n(2 n-1)}\left(1-q^{2 j+1}\right)^{2}\left(1+q^{2 j+1}\right) \\
& =\frac{\left(q^{2} ; q^{2}\right)_{\infty}}{\left(q ; q^{2}\right)_{\infty}} \sum_{n=0}^{\infty} \frac{(-1)^{n} q^{n(n-1)}}{\left(q^{2} ; q^{2}\right)_{n}}\left(1-q^{2 n+1}\right) \\
& =\frac{\left(q^{2} ; q^{2}\right)_{\infty}}{\left(q ; q^{2}\right)_{\infty}}\left(\sum_{n=0}^{\infty} \frac{(-1)^{n} q^{n(n-1)}}{\left(q^{2} ; q^{2}\right)_{n}}-q \sum_{n=0}^{\infty} \frac{(-1)^{n} q^{n(n+1)}}{\left(q^{2} ; q^{2}\right)_{n}}\right)=-q \frac{\left(q^{2} ; q^{2}\right)_{\infty}^{2}}{\left(q ; q^{2}\right)_{\infty}}
\end{aligned}
$$

For (32) we consider the Bailey pair (25) with the conjugate Bailey pair (14) with $u_{n}=v_{n}=1 /\left(q^{2} ; q^{2}\right)_{n}$. Our result follows from applying (24).

\section{Corollary 5.7.}

$$
2 \sum_{j \geq 2|n|}(-1)^{j} q^{j(j+1)-n(2 n-1)}=\left(q^{2} ; q^{2}\right)_{\infty}\left((-q)_{\infty}+(q)_{\infty}\right),
$$

$$
2 \sum_{j \geq 2|n|}(-1)^{n} q^{j(3 j+2)-2 n(3 n-1)}\left(1-q^{2 j+1}\right)=\left(q^{4} ; q^{4}\right)_{\infty}+(q)_{\infty}^{2}\left(-q^{2} ; q^{2}\right)_{\infty}
$$

$$
\begin{aligned}
2 \sum_{j \geq 2|n|}(-1)^{n} q^{3 j^{2}-2 n(3 n-1)}\left(1+q^{2 j+1}\right)^{2}\left(1-q^{2 j+1}\right)= & (2+q)\left(q^{4} ; q^{4}\right)_{\infty} \\
& +q(q)_{\infty}^{2}\left(-q^{2} ; q^{2}\right)_{\infty}
\end{aligned}
$$

Proof. For (33), we consider the Bailey pair (22) with the conjugate Bailey pair (13) with $u_{n}=v_{n}=1 /\left(q^{2} ; q^{2}\right)_{n}$. Our result follows from applying (24):

$$
\begin{aligned}
\sum_{j \geq 2|n|}(-1)^{j} q^{j(j+1)-n(2 n-1)} & =\left(q^{2} ; q^{2}\right)_{\infty} \sum_{n=0}^{\infty} \frac{q^{n(2 n+1)}}{(q)_{2 n}} \\
& =\frac{\left(q^{2} ; q^{2}\right)_{\infty}}{2} \sum_{n=0}^{\infty} \frac{\left(1+(-1)^{n}\right) q^{n(n+1) / 2}}{(q)_{n}} \\
& =\frac{\left(q^{2} ; q^{2}\right)_{\infty}\left((q)_{\infty}+(-q)_{\infty}\right)}{2}
\end{aligned}
$$

For (34), we consider the Bailey pair (27) with the conjugate Bailey pair (8) with $q \rightarrow-q$ and $u_{n}=v_{n}=1 /\left(q^{2} ; q^{2}\right)_{n}$. We then apply (24) as before. 
For (35), we consider the Bailey pair (27) with the conjugate Bailey pair (9) with $q \rightarrow-q$ and $u_{n}=v_{n}=1 /\left(q^{2} ; q^{2}\right)_{n}$. Our result follows from applying (24):

$$
\begin{aligned}
\sum_{j \geq 2|n|}(-1)^{n} q^{3 j^{2}-2 n(3 n-1)}\left(1+q^{2 j+1}\right)^{2}\left(1-q^{2 j+1}\right) & \\
& =\frac{\left(q^{2} ; q^{2}\right)_{\infty}}{\left(-q ; q^{2}\right)_{\infty}} \sum_{n=0}^{\infty} \frac{\left(1+q^{2 n+1}\right) q^{n(2 n-1)}}{(q)_{2 n}} \\
& =(q)_{\infty}\left(-q^{2} ; q^{2}\right)_{\infty}\left(\sum_{n=0}^{\infty} \frac{q^{n(2 n-1)}}{(q)_{2 n}}+q \sum_{n=0}^{\infty} \frac{q^{n(2 n+1)}}{(q)_{2 n}}\right) \\
& =\left(1+\frac{q}{2}\right)\left(q^{4} ; q^{4}\right)_{\infty}+\frac{(q)_{\infty}^{2}\left(-q^{2} ; q^{2}\right)_{\infty}}{2} .
\end{aligned}
$$

\section{Applications to partitions}

In this section we present some partition identities. We define a partition as a finite nonincreasing sequence of positive integers, $\lambda=\left(\lambda_{1}, \ldots, \lambda_{k}\right)$. We refer to each $\lambda_{i}$ as a part of the partition. We say that $\lambda$ is a partition of $n$, or $|\lambda|=n$, if the sum of its parts is equal to $n$. For example, there are 7 partitions of 5:
$(5)$,
$(4,1)$,
$(3,2)$,
$(3,1,1)$,
$(2,2,1)$
$(2,1,1,1)$
$(1,1,1,1,1)$.

We say that a partition $\lambda$ is without gaps if for every positive integer $k<\lambda_{1}$, there exists $i$ such that $\lambda_{i}=k$. As in the last section, we can generalize this notion to without odd/even gaps by restricting $k$ to odd/even positive integers.

Theorem 6.1. Let $a(n)$ be the number of partitions of $n$ with largest part odd and without odd gaps. Let $b(n)$ be the number of partitions of $n$ into parts congruent to $\pm 1, \pm 3, \pm 5, \pm 7, \pm 8, \pm 9 \bmod 20$. Then $a(n)=b(n-1)$ for $n \geq 1$.

Proof. We consider the Bailey pair [Slater 1951, C(1)]

$$
\alpha_{2 n}=(-1)^{n} q^{2 n(3 n+1)}, \alpha_{2 n+1}=0, \quad \beta_{n}=1 /\left(q^{2} ; q^{4}\right)_{n}\left(q^{2} ; q^{2}\right)_{n}
$$

with the conjugate Bailey pair (5) (note that $q \rightarrow-q$ ) with $u_{n}=v_{n}=1 /\left(q^{2} ; q^{2}\right)_{n}$ to get

$$
\begin{aligned}
\frac{(q)_{\infty}}{\left(q^{2} ; q^{4}\right)_{\infty}} \sum_{n=0}^{\infty} \frac{q^{n(n+2)}}{(q)_{2 n+1}} & =\sum_{n=-\infty}^{\infty}(-1)^{n} q^{2 n(n+1)} \sum_{j \geq 2|n|}(-1)^{j} q^{2 j(j+1)} \\
& =\sum_{j=-\infty}^{\infty}(-1)^{j} q^{2 j(5 j+3)}=\left(q^{4}, q^{16}, q^{20} ; q^{20}\right)_{\infty}
\end{aligned}
$$


where the last equality follows from the Jacobi triple product; see [Andrews 1998, Theorem 2.8]. It is left to note that

$$
\begin{aligned}
& q \sum_{n=0}^{\infty} \frac{q^{n(n+2)}}{(q)_{2 n+1}}=\sum_{n=0}^{\infty} \frac{q^{1+3+\cdots+(2 n+1)}}{(1-q)\left(1-q^{2}\right) \cdots\left(1-q^{2 n+1}\right)}
\end{aligned}
$$

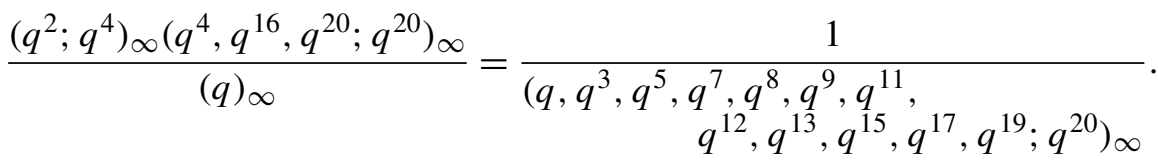

We can also define a weight function $\omega(\lambda)$ for a partition. If we say that $a(n)$ counts the number of partitions of $n$ with respect to the weight function $\omega(\lambda)$ then $a(n)=\sum_{|\lambda|=n} \omega(\lambda)$.

Theorem 6.2. Let $g(n)$ be the number of partitions of $n$ without even gaps and with corresponding weight function $\omega(\lambda)=(-1)^{k_{1}} 2^{k_{2}}$, where $k_{1}$ is the multiplicity of the largest part plus the number of even parts less than the largest part and $k_{2}$ is the number of different odd parts less than the largest part. Then

$$
g(n)= \begin{cases}(-1)^{j}(2 j+1) & \text { if } n=j(2 j+1) \text { for } j \in \mathbb{N}, \\ (-1)^{j+1}(2 j+1) & \text { if } n=(j+1)(2 j+1) \text { for } j \in \mathbb{N}, \\ 0 & \text { otherwise. }\end{cases}
$$

Example 6.3. Consider $n=8$ and $n=10$ :

\begin{tabular}{lrlr}
$|\lambda|=8$ & $\omega(\lambda)$ & $|\lambda|=10$ & $\omega(\lambda)$ \\
\hline$(4,2,2)$ & -1 & $(4,4,2)$ & -1 \\
$(4,2,1,1)$ & 2 & $(4,3,2,1)$ & 4 \\
$(3,3,2)$ & -1 & $(4,2,2,2)$ & 1 \\
$(3,2,2,1)$ & -2 & $(4,2,2,1,1)$ & -2 \\
$(3,2,1,1,1)$ & 2 & $(4,2,1,1,1,1)$ & 2 \\
$(2,2,2,2,2)$ & -1 & $(3,3,2,2)$ & 1 \\
$(2,2,2,2,1,1)$ & 2 & $(3,3,2,1,1)$ & -2 \\
$(2,2,2,1,1,1,1)$ & -2 & $(3,2,2,2,1)$ & 2 \\
$(2,2,1,1,1,1,1,1)$ & 2 & $(3,2,2,1,1,1)$ & -2 \\
$(2,1,1,1,1,1,1,1,1)$ & -2 & $(3,2,1,1,1,1,1)$ & 2 \\
$(1,1,1,1,1,1,1,1,1,1)$ & 1 & $(2,2,2,2,2)$ & -1 \\
& & $(2,2,2,2,1,1)$ & 2 \\
& & $(2,2,2,1,1,1,1)$ & -2 \\
& & $(2,2,1,1,1,1,1,1)$ & 2 \\
& & $(2,1,1,1,1,1,1,1,1)$ & -2 \\
& & $(1,1,1,1,1,1,1,1,1,1)$ & 1 \\
\hline & 0 & & 5
\end{tabular}


Proof. We consider the Bailey pair (21) and the conjugate Bailey pair (15) with $u_{n}=v_{n}=1 /\left(q^{2} ; q^{2}\right)_{n}$ to get

$$
\sum_{j=0}^{\infty}(2 j+1)\left(1-q^{2 j+1}\right)(-1)^{j} q^{j(2 j+1)}=\sum_{n=0}^{\infty} \frac{\left(-q ; q^{2}\right)_{n}(-1)^{n} q^{n(n+1)}}{(q ;-q)_{2 n}\left(1+q^{2 n+1}\right)} .
$$

We then note that

$$
\begin{aligned}
& \sum_{n=0}^{\infty} \frac{\left(q ; q^{2}\right)_{n}(-1)^{n} q^{n(n+1)}}{(q ;-q)_{2 n}\left(1+q^{2 n+1}\right)} \\
& =\sum_{n=0}^{\infty}\left(1+2 q+2 q^{2}+\cdots\right)\left(1+2 q^{3}+2 q^{6}+\cdots\right) \cdots\left(1+2 q^{2 n-1}+2 q^{4 n-2}+\cdots\right) \\
& \quad \times\left(1-q^{2 n+1}+q^{4 n+2}-\cdots\right) \cdot \frac{(-1)^{n} q^{2+4+\cdots+2 n}}{\left(1+q^{2}\right)\left(1+q^{4}\right) \cdots\left(1+q^{2 n}\right)} \cdot \quad
\end{aligned}
$$

Theorem 6.4. Let a $(n)$ denote the number of partitions of $n$ without even gaps and having an even number of parts, minus the number of partitions of $n$ without even gaps and having an odd number of parts. Then $a(0)=1$ and for $n>0$,

$$
a(n)= \begin{cases}1 & \text { if } n=j(5 j+3) / 2 \text { for } j \in \mathbb{N}, \\ -1 & \text { if } n=j(5 j-3) / 2 \text { for } j \in \mathbb{N}, \\ 0 & \text { otherwise. }\end{cases}
$$

Proof. Consider the Bailey pair

$$
\alpha_{n}=(-1)^{n} q^{n(3 n+1) / 2}, \quad \beta_{n}=\frac{\left(q ; q^{2}\right)_{n}}{\left(q^{2} ; q^{2}\right)_{2 n}} .
$$

This follows from specializing [Bailey 1948, page 5, Section 6, (ii)] with $a=1$, $b \rightarrow \infty$ and with $x$ replaced by $q$, together with the conjugate Bailey pair (15) with $u_{n}=v_{n}=1 /\left(q^{2} ; q^{2}\right)_{n}$. Then we get

$$
\begin{aligned}
\sum_{j=0}^{\infty}\left(1-q^{2 j+1}\right) q^{j(5 j+3) / 2} & =\sum_{n=0}^{\infty} \frac{(-1)^{n} q^{n(n+1)}}{(-q)_{2 n+1}} \\
& =\sum_{n=0}^{\infty} \frac{(-1)^{n} q^{2+4+\cdots+2 n}}{(1+q)\left(1+q^{2}\right) \cdots\left(1+q^{2 n+1}\right)} .
\end{aligned}
$$

\section{Conclusions and future work}

This work hopes to bridge the gap between the work done by Bailey and Slater and the work done recently by Andrews and Warnaar. We can now clearly see how our new conjugate Bailey pairs relate to those introduced over 50 years ago by Bailey. 
We recall the reference to WP Bailey pairs and chains. As with the large amount of work done on Bailey chains, it is hoped and anticipated that these chains do appear for conjugate Bailey pairs as well.

We note that we have focused on applications to triangular numbers, indefinite quadratic forms and partitions, but we are not limited to these. Future work may address weighted $q$-series identities, generalized Lambert series, Ramanujan-type identities and a more thorough treatment of partition identities. It should also be noted that a combinatorial proof of any of the partition identities would also be desirable as it might provide more insight into similar identities.

We have only defined 15 new conjugate Bailey pairs from Corollary 3.1, though infinitely many exist. Using Corollary 3.2, we could also define analogous conjugate Bailey pairs with respect to the asymmetric bilateral Bailey transform, which would then lead to new results similar to those presented above.

\section{Acknowledgments}

The author would like to acknowledge and thank George Andrews and the reviewer for the helpful suggestions.

\section{References}

[Andrews 1984] G. E. Andrews, "Hecke modular forms and the Kac-Peterson identities", Trans. Amer. Math. Soc. 283:2 (1984), 451-458. MR 85e:11031 Zbl 0545.10016

[Andrews 1986a] G. E. Andrews, "The fifth and seventh order mock theta functions", Trans. Amer. Math. Soc. 293:1 (1986), 113-134. MR 87f:33011 Zbl 0593.10018

[Andrews 1986b] G. E. Andrews, "Ramanujan's "lost” notebook, V: Euler's partition identity", Adv. in Math. 61:2 (1986), 156-164. MR 87i:11137 Zbl 0601.10007

[Andrews 1998] G. E. Andrews, The theory of partitions, Cambridge University Press, Cambridge, 1998. MR 99c:11126 Zbl 0996.11002

[Andrews 2001] G. E. Andrews, "Bailey's transform, lemma, chains and tree", pp. 1-22 in Special functions 2000: Current perspective and future directions (Tempe, AZ), edited by J. Bustoz et al., NATO Sci. Ser. II Math. Phys. Chem. 30, Kluwer, Dordrecht, 2001. MR 2004i:33027 Zbl 1005.33005

[Andrews and Warnaar 2007] G. E. Andrews and S. O. Warnaar, "The Bailey transform and false theta functions”, Ramanujan J. 14:1 (2007), 173-188. MR 2008a:33019 Zbl 1116.33017

[Andrews et al. 1988] G. E. Andrews, F. J. Dyson, and D. Hickerson, "Partitions and indefinite quadratic forms", Invent. Math. 91:3 (1988), 391-407. MR 89f:11071

[Bailey 1948] W. N. Bailey, "Identities of the Rogers-Ramanujan type", Proc. London Math. Soc.

(2) 50 (1948), 1-10. MR 9,585b Zbl 0031.39203

[Berndt 1991] B. C. Berndt, Ramanujan's notebooks, III, Springer, New York, 1991. MR 92j:01069 Zbl 0733.11001

[Dickson 1966] L. E. Dickson, History of the theory of numbers, II: Diophantine analysis, Chelsea, New York, 1966. MR 39 \#6807b Zbl 0958.11500 
[Gasper and Rahman 2004] G. Gasper and M. Rahman, Basic hypergeometric series, 2nd ed., Encyclopedia of Mathematics and its Applications 96, Cambridge University Press, Cambridge, 2004. MR 2006d:33028 Zbl 1129.33005

[Hecke 1959] E. Hecke, "Über einen Zusammenhang zwischen elliptischen Modulfunktionen und indefiniten quadratischen Formen", pp. 418-427 in Mathematische Werke, Vandenhoeck und Ruprecht, Göttingen, 1959. MR 21 \#3303 Zbl 0092.00102

[Rogers 1894] L. J. Rogers, "Second memoir on the expansion of certain infinite products", Proc. London Math. Soc. 25 (1894), 318-343.

[Slater 1951] L. J. Slater, “A new proof of Rogers's transformations of infinite series", Proc. London Math. Soc. (2) 53 (1951), 460-475. MR 13,227g Zbl 0044.06102

[Slater 1952] L. J. Slater, "Further identies of the Rogers-Ramanujan type", Proc. London Math. Soc. (2) 54 (1952), 147-167. MR 14,138e Zbl 0046.27204

Received February 19, 2008. Revised June 26, 2008.

Michael J. ROWELL

DePartment of MATHEMATICS AND COMPUter SCIENCE

PACIFIC UNIVERSITY

FOREST GROVE, OR 97116

UNITED STATES

rowell@pacificu.edu

www.math.pacificu.edu/ rowell 